\title{
STUDY OF THE EFFECT OF BRANCHING IN DEGRADATION OF POLYETHYLENES OBTAINED VIA METALLOCENE CATALYST
}

\author{
MARIO ESCUDERO ACEVEDO ${ }^{A *}$, RAÚL QUIJADA $^{A}$, MARCELO CAMPOS VALLETTE $^{B}$
}

\author{
a Departamento de Ingeniería Química y Biotecnología, Facultad de Ciencias Físicas y Matemáticas, Universidad de Chile and Centro para la Investigación \\ Interdisciplinaria Avanzada en Ciencias de los Materiales (CIMAT), Casilla 2777, Santiago, Chile. \\ ${ }^{b}$ Departamento de Química, Facultad de Ciencias, Universidad de Chile, Casilla 653, Santiago, Chile. \\ (Received: 21 January 2007 - Accepted: 17 March 2008)
}

\begin{abstract}
Polyethylenes, homo and copolymers obtained via metallocene catalyst, were synthesized and used to study the effect of comonomer size and content in the main linear backbone polymer on its degradation. Homoethylene metallocene polymer, ethylene-1-hexene and ethylene-1-octadecene metallocene copolymer films were characterized and analyzed during their aging in a forced draft oven at $60{ }^{\circ} \mathrm{C}$ for one year. The polymers were characterized before and after ageing using GPC, DSC, FTIR, and tensile tests in order to detect changes in chemical structure, size, and molecular weight distribution as well as to quantify the effect of time over their useful life. As a qualitative reference parameter, the carbonyl index, the ratio of the infrared absorbance of the $\mathrm{CO}$ stretching band at $1715 \mathrm{~cm}^{-1}$ and the absorbance of a reference band at $718 \mathrm{~cm}^{-1}$, was determined. Results showed that kinetic thermooxidation is related to comonomer size and content in the main backbone polymer. As comonomer size decreases or comonomer content increases, degradation rate increases. This can be observed through the scission factor (S) and carbonyl index (CI) graphs of each material which show a slope increasing related to the autocatalytic rate of oxidation.
\end{abstract}

Keywords: Thermal oxidation, Degradation, Metallocene, Polyethylene, 1-Olefine, Copolymers.

\section{INTRODUCTION}

The advent of metallocene catalysts has made possible the production of various tailor-made polymers ${ }^{1-4}$ to satisfy industrial and end user requirements. This is due to the fact that metallocene catalysts are single-site catalysts that lead to narrower molecular weight distribution and more uniform structure than traditional Ziegler-Natta catalysts. The use of this new family of catalysts has allowed the synthesis of polyolefins with added value with respect to traditional commodity production of polyethylene (HDPE and LDPE). In particular, the copolymerization of ethylene with higher $\alpha$-olefins has gaven rise to linear low density polyethylene (LLDPE), yielding copolymers with homogenous comonomer content ${ }^{5,6}$.

Metallocene linear low density polyethylenes (mLLDPE) are characterized by their high resistance and tenacity compared to other thermoplastics. They are used mostly for packaging and in greenhouses (with mulching films as the new trend), standing out because of their lightness, inertness and durability. Their accumulation, however, causes environmental pollution since process degradation is low.

The study of their degradation is an area of continuous development ${ }^{7-10}$. Thermal degradation of polymers can be carried out in an inert environment (pyrolisis) or in an oxidative environment (air), and it is described by a random scission mechanism which does not follow first order degradation kinetics ${ }^{10}$. Reich and Stivala ${ }^{11}$ describe that during the degradation process inititators are formed (alkyl and peroxyl radicals) in an autocatalytic degradation reaction, scissioning the macromolecule into fragments of lower molecular weight, and thereby affecting the material's mechanical properties ${ }^{12}$. Therefore the need to study the influence of 1-olefin comonomer type and content in the thermal oxidation of metallocene ethylene-1-olefin copolymer films arises naturally.

\section{EXPERIMENTAL}

Reagents, materials and methods.

Rac-ethylenebis(indenyl)zirconiumdichloride $\quad\left(\mathrm{Et}(\mathrm{Ind})_{2} \mathrm{ZrCl}_{2}\right) \quad$ catalyst from Crompton Corporation (Bergkamen, Germany) and methylaluminoxane cocatalyst (MAO) at $10 \mathrm{vol} \%$ in toluene from Witco GMBH (Bergkamen, Germany) were used for the polymerization reaction. Ethylene $>99.99 \%$ purity from AGA S.A. (Chile) was purified by passing the gas through 2 columns of $4 \AA$ molecular sieves to remove traces of water, oxygen and sulfur. Toluene solvent from J.T. Baker (Xalostoc, Mexico) and comonomers 1-hexene and 1 -octadecene from Aldrich (Milwaukee, WI, USA) were purified by refluxing for 3 hours over metallic sodium and then it was proceeded to distill. Catalyst and cocatalyst were handled under a strong nitrogen stream.

Synthesized homoethylene polymer and ethylene-1-hexene and ethylene1-octadecene metallocene copolymers were obtained as powder at the Laboratorio de Ingeniería de Polímeros, Universidad de Chile.

Polymerization.

Homoethylene polymer and ethylene-1-olefin copolymers with different comonomer size (1-hexene and 1-octadecene) and content were synthesized by coordination polymerization using the metallocene catalyst rac-ethylenebis (indenyl)zirconium dichloride (Et(Ind) ${ }_{2} \mathrm{ZrCl}_{2}$ ) and methylaluminoxane (MAO) as cocatalyst.

The polymerization reaction was carried out in a $1 \mathrm{~L}$ Parr reactor previously heated to $150{ }^{\circ} \mathrm{C}$ for 2 hours to eliminate traces of water. Once in operation, nitrogen purges were made to eliminate air from the reactor. Toluene $(500 \mathrm{~mL})$ was added to the reactor in a nitrogen environment and reactor was warmed to $60{ }^{\circ} \mathrm{C}$, adding MAO cocatalyst in a $\mathrm{Al} / \mathrm{Zr}$ ratio of $6 \times 10^{3}$ under a flow of nitrogen, with constant stirring at $500 \mathrm{rpm}$. Once the nitrogen flow was stopped, ethylene ( 2 bar pressure) and comonomer in the case of copolymer polymerization were introduced, adding metallocene catalyst at a concentration of $3.5 \times 10^{-7} \mathrm{~mol} / \mathrm{L}$, keeping the reaction going for $30 \mathrm{~min}$. The polymerization reactions were terminated by addition of a $2 \mathrm{vol} \% \mathrm{HCl} / \mathrm{CH}_{3} \mathrm{OH}$ solution. The synthesized polymers were precipitated from the reaction medium, washed several times with cold ethanol, and dried for one day at room temperature.

Polymer Characterization. tests.

Polymers were characterized by GPC, NMR, FTIR, DSC and tensile

Molar comonomer content (\%) in the main copolymer backbone was measured by ${ }^{13} \mathrm{CNMR}$ at $120^{\circ} \mathrm{C}$ on a Varian Inova XLS 300 spectrometer at 75 MHz. Samples were prepared by dissolving the polymer in o-dichlorobenzene and benzene- $\mathrm{d}_{6}(20 \% \mathrm{v} / \mathrm{v})$ in $5 \mathrm{~mm}$ tubes. Chromium(III) tris(acetylacetonate) was used as paramagnetic substance to reduce relaxation times. The results are shown in Table 1 . The nomenclature used to identify the metallocene homopolymer and copolymers are "mPE", "mPE-H\#" and "mPE-O\#”, where $\mathrm{mPE}$ refers to linear metallocene ethylene homopolymer, $\mathrm{mPE}-\mathrm{H}$ refers to metallocene ethylene-1-hexene copolymers, mPE-O to ethylene-1-octadecene copolymers, and \# is the molar comonomer content (\%) in the main copolymer backbone.

Molecular weight was measured by gel permeation chromatography (GPC) on a Waters Alliance 2000 apparatus with a differential refractive index detector. The mobile phase was 1,2,4-trichlorobenzene to which 2,6di-tert-butyl-4-methyl phenol (BHT) antioxidant $(0.1 \%)$ was added. The analytical flow rate was $1 \mathrm{~mL} / \mathrm{min}$ and the temperature was $140^{\circ} \mathrm{C}$. The values obtained were referenced to a universal curve with the following viscosimetric parameters $\mathrm{K}=3.95 \times 10^{-4}$ and $\alpha=0.726$ (polyethylene).

Melting temperature and melting heat were measured by differential scanning calorimetry on a TA Instruments DSC 2920 apparatus connected to a cooling system calibrated with different standards. The heating/cooling ramp rates were $10^{\circ} \mathrm{C} / \mathrm{min}$ in an inert atmosphere of $\mathrm{N}_{2}$ flowing at $80 \mathrm{~mL} / \mathrm{min}$ from ambient temperature to $180^{\circ} \mathrm{C}$. To erase the thermal history of the material, first a heating ramp rate of $20^{\circ} \mathrm{C} / \mathrm{min}$ was used, followed by a cooling ramp and a consecutive heating ramp of $10{ }^{\circ} \mathrm{C} / \mathrm{min}$ each. The data gathered are from the second and third ramp. A value of $290 \mathrm{~J} / \mathrm{g}$ has been taken as the enthalpy of a perfect polyethylene crystal in order to calculate crystallinities from the melting enthalpies. 
Chemical structures were determined from the FTIR spectra on a Bruker Vector 22 spectrometer. The spectra were accumulated 4 times between 4000 and $400 \mathrm{~cm}^{-1}$ with a resolution of $4 \mathrm{~cm}^{-1}$.

Tensile strength and Young's modulus were obtained on an HP D500 tensile stress dynamometer. Five samples were tested for each material (ca. $0.3 \mathrm{~mm}$ thick, $5 \mathrm{~mm}$ wide, $80 \mathrm{~mm}$ long, and $30 \mathrm{~mm}$ grip-to-grip length) at 23 ${ }^{\circ} \mathrm{C}, 32 \%$ relative humidity, and a displacement rate of $50 \mathrm{~mm} / \mathrm{min}$. Young's modulus was obtained from the slope of the stress-strain curve (linear curve zone), and tensile strength was obtained at the yield point where the material suffers permanent strain once the stress is removed. The method of tangents for determining stress, where the tangents to the initial and final parts of the loadelongation curve intersect, was used in those cases in which the yield point could not be distinguished ${ }^{13}$.

Polymer Thermal Oxidation.

Polymer films were aged for one year in a forced draft oven (MEMMERT, model ULM400, vol. 53 l) at $60^{\circ} \mathrm{C}$ with ca. $3 \mathrm{~m} / \mathrm{s}$ air flow near to the fan center measured by Airflow anemometer model LCA 6000 .

Powders were placed in a $10 \mathrm{~cm} \times 10 \mathrm{~cm} \times 0.1 \mathrm{~mm}$ steel strap frame between two $0.4 \mathrm{~mm}$ thick teflon sheets, and press-mould for $3 \mathrm{~min}$ at $170{ }^{\circ} \mathrm{C}$ and 40 bar in a hydraulic press (Industria HP). Two films of each polymer to be aged were placed separately in the oven so that a homogeneous air stream would go through them.

One film was monitored during the aging process, cutting some pieces for characterization by FTIR and GPC. The other was tested mechanically for tensile strength once the aging process was finished.

In the FTIR method the carbonyl group peak was measured between 1715 and $1720 \mathrm{~cm}^{-114}$. As a qualitative parameter, the carbonyl index was determined as the ratio between the $\mathrm{CO}$ stretching band peak intensity at $1715 \mathrm{~cm}^{-1}$ and the peak intensity of a reference band at $718 \mathrm{~cm}^{-1}$, corresponding to methylene rocking vibrations. The oxidation rates of the materials were compared from the slope of the carbonyl index vs. aging time graphs.

For the GPC determination, weight-average molecular weight (Mw) and number-average molecular weight $(\mathrm{Mn})$ were measured to account for size evolution and polymer chain distribution.

\section{RESULTS AND DISCUSSIONS}

Metallocene Synthesis.

Table 1 presents the properties of metallocene polymers which exhibit a lower polydispersity close to 2 , which means they have a narrower molecular weight distribution. In the literature it is known that comonomer (branch) size and content have an influence on the material's crystallization, melting temperature and crystallinity ${ }^{15}$. This is shown in our results. It is seen that for a given comonomer size, as its content in the linear backbone chain (mole $\%$ ) increases, the polymer's melting temperature decreases. Branching inserted into the linear polymer's backbone inhibits the sliding movement of the chain to form an ideal folded structure (chain folding), producing a shorter folding length than that with no branches, and therefore decreasing crystal size (lamellar thickness). Melting temperature and lamellar crystal thickness are correlated by the Gibbs-Thomson equation. Due to the decrease in lamellar crystal thickness, there is an increase of the amorphous phase in the polymer.

Table 1. Properties of metallocene polymers.

\begin{tabular}{lcccccc}
\hline \multicolumn{1}{c}{ Films } & Comonomer & Melting & $\mathbf{M w} \cdot \mathbf{1 0}^{-\mathbf{3}}$ & $\mathbf{M n \cdot 1 0 ^ { - 3 }}$ & Mw/Mn & Cristallinity \\
\hline \multicolumn{1}{c}{ Code } & {$[\%]$} & {$\left[{ }^{\circ} \mathbf{C}\right]$} & {$[\mathbf{k g} / \mathbf{k m o l}]$} & {$[\mathbf{k g} / \mathbf{k m o l ]}$} & {$[$ Adim] } & {$[\%]$} \\
\hline mPE & - & 136 & 116 & 64 & 1.8 & 68 \\
mPE-H7.8 & 7.8 & 88 & 69 & 41 & 1.7 & 6 \\
mPE-H14.1 & 14.1 & 69 & 67 & 41 & 1.6 & 3 \\
mPE-07.1 & 7.1 & 92 & 95 & 55 & 1.7 & 4 \\
\hline
\end{tabular}

Molecular Weight and Infrared Spectroscopy

It is seen in Table 2 that metallocene polymer films undergo changes in their molecular weight after one year of accelerated aging.
Table 2. Number average molecular weight (Mn) of metallocene polymer films aged in a forced draft oven at $60{ }^{\circ} \mathrm{C}$ for one year.

\begin{tabular}{ccccc}
\hline \multicolumn{5}{c}{ Mn· 10-3 [kg/kmol] } \\
\hline Aged [h] & mPE & mPE H7.8 & mPE-H14.1 & mPE-07.1 \\
\hline 0 & 64 & 41 & 41 & 55 \\
756 & 34 & 41 & 33 & 42 \\
1432 & 28 & 42 & 36 & 43 \\
1649 & 26 & 40 & 35 & 46 \\
1839 & 23 & 40 & 36 & 49 \\
2435 & 21 & 37 & 35 & 47 \\
4888 & 13 & 35 & 27 & 48 \\
9136 & 11 & 11 & 5 & 22 \\
\hline
\end{tabular}

The degradation process occurs by random chain scission, reducing chain length and thereby shifting the molecular weight distribution to lower values ${ }^{16}$. Then those scission reactions have a clear influence on the number-average molecular weight.

One way to visualize the influence of scission reactions over number average molecular weight $(\mathrm{Mn})$ is by means of the scission factor $(\mathrm{S})$, which is defined as the apparent number of chain scissions per molecule:

$$
S=\left(M_{m o} / M_{n}\right)-1
$$

where $M_{n o}$ and $M_{n t}$ are the number average molecular weights at time zero and time t, respectively whose data are obtained from Table 2 and later plotted in Figure 1, which represents the molecular scission underwent by polymers aged, it is seen that metallocene polyethylene degrades earlier than the other polymers.

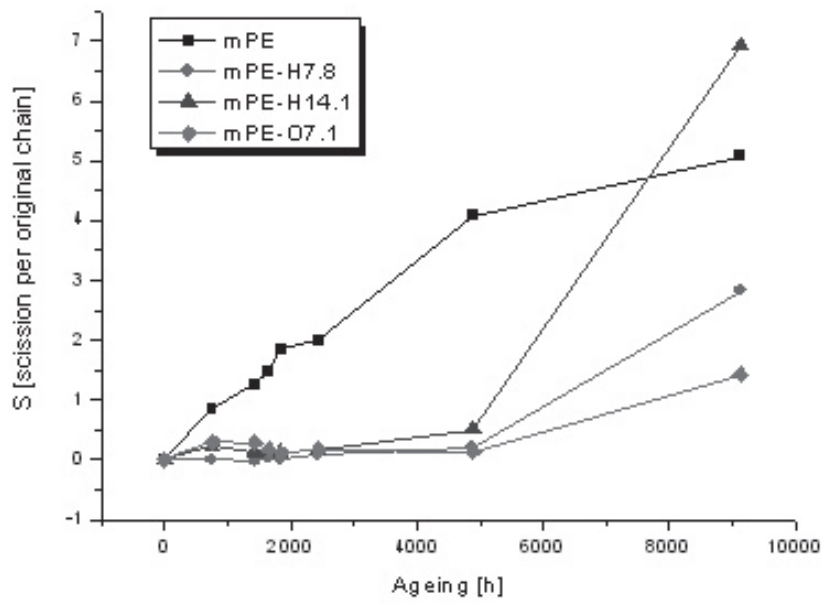

Figure 1. Scission factor for metallocene polymers aged in a forced draft oven at $60{ }^{\circ} \mathrm{C}$ for one year.

In general, linear chain polymers such as metallocene polyethylene are more prone to rupture than coiled chain polymers represented by metallocene ethylene-1-olefin copolymers. An increase in branch length induces a mainly globular polymer conformation. If this really happens, a degradation rate delay should be expected during polymer aging. In fact, this can be deduced from Figures 1 and 2, where Figure 2 represents the influence of branch length on the ratio of degradation for two types of polymers (1-hexene and 1-octadecene copolymers), trend shown by the carbonyl index graphs, i.e. growth of carbonyl group by polymer oxidation. 


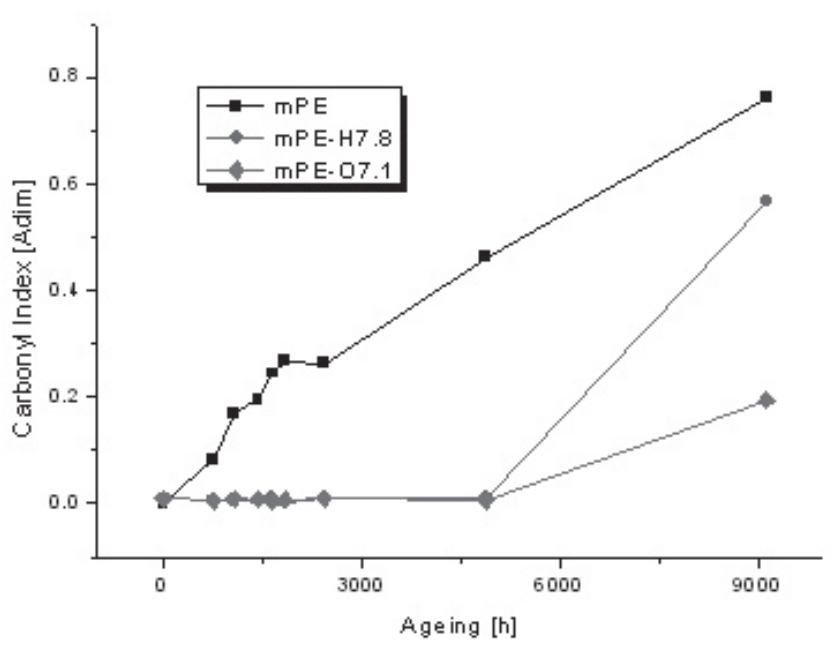

Figure 2. Carbonyl Index graph for metallocene homoethylene and ethylene-1-olefin copolymers in a forced draft oven at $60{ }^{\circ} \mathrm{C}$ for one year.

The scission graph (Figure 1)shows that the degradation rate of the copolymers starts increasing after $4880 \mathrm{~h}$ of aging, representing an increase in autocatalytic degradation ${ }^{17}$, close to the trend shown by the carbonyl index graphs of all the polymers studied, which are represented in Figure 3. This figure shows growth of carbonyl group in homo and ethylene copolymers due to polymer thermal oxidation. This confirms the idea of degradation rate delay.

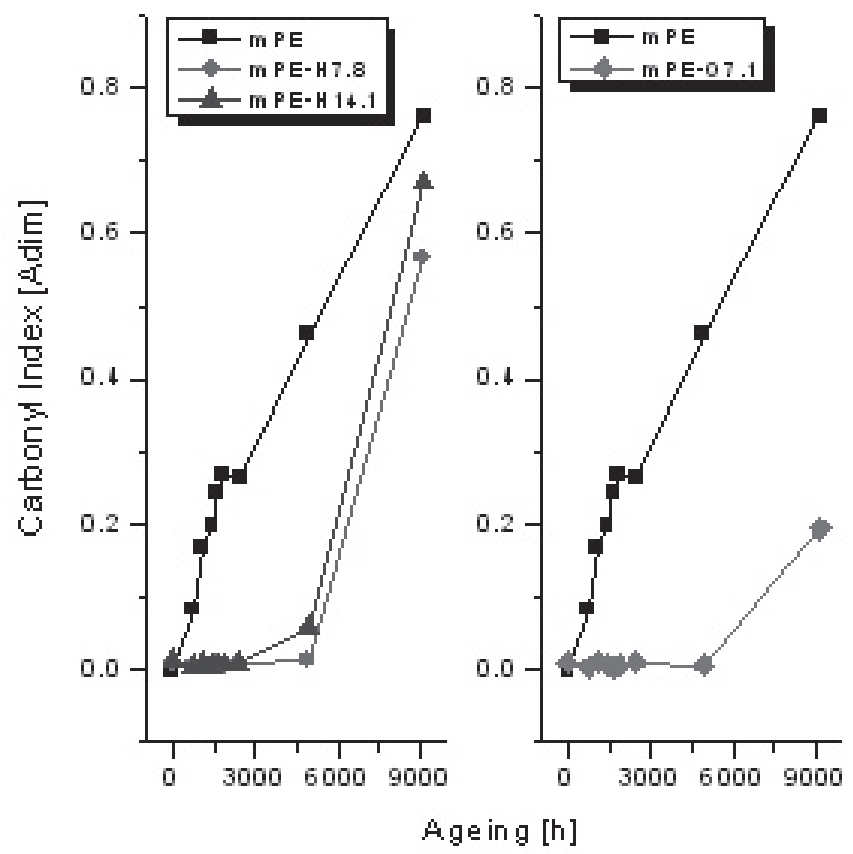

Figure 3. Carbonyl index graph for metallocene polymers aged in a forced draft oven at $60^{\circ} \mathrm{C}$ for one year.

On the other hand, from Figures 1 and 3 (1-hexene ethylene copolymer) it is possible to deduce that when the molar content of comonomer (branch) increases, degradation rate increases. One possible explanation would be that when comonomer content increases in the main chain, the terminal hydrogens of tertiary carbons, whose bonds are more labile than those of secondary hydrogens, would also increase. When the polymer undergoes an external thermal action, radical concentration could increase by an intramolecular scission mechanism; then, radicals could catalyze the chain degradation by means of an intermolecular scission mechanism. This step determines the degradation rate.

Mechanical Tensile Parameters.

In Table 3 are presented the mechanical tensile parameters for metallocene polymers.

Table 3. Mechanical parameters obtained from tensile tests of polymer films without $(0 \mathrm{~h})$ and with aging $(9136 \mathrm{~h})$ in a forced draft oven at $60{ }^{\circ} \mathrm{C}$ (average values).

\begin{tabular}{|c|c|c|c|c|c|c|c|c|}
\hline \multirow[b]{2}{*}{ Films } & \multicolumn{4}{|c|}{0 [h] } & \multicolumn{4}{|c|}{$9136[\mathrm{~h}]$} \\
\hline & E & $\sigma$ yield & $\varepsilon$ break & o_break & $\mathrm{E}$ & $\sigma$ yield & $\varepsilon$ break & $\sigma$ break \\
\hline Code & [Mpa] & [Mpa] & {$[\%]$} & [Mpa] & [Mpa] & [Mpa] & {$[\%]$} & [Mpa] \\
\hline $\mathrm{mPE}$ & 648 & 26 & 596 & 27 & $x$ & $x$ & $x$ & $x$ \\
\hline mPE-H7.8 & 41 & 3 & 959 & 28 & 38 & 3 & 141 & 5 \\
\hline mPE-H14.1 & 7 & 1 & 1407 & 14 & 4 & 1 & 432 & 4 \\
\hline mPE-07.1 & 48 & 3 & 708 & 21 & 44 & 3 & 337 & 10 \\
\hline
\end{tabular}

${ }^{x}$ No data exist because of material's fragility.

According to Li Pi Shan et al. and Dilara et al. ${ }^{18,19}$, tensile strength at yield $\left(\sigma_{\text {yield }}\right)$ increases when the material's percentage crystallinity increases. In Table 3 it is seen that tensile strength at yield values of the most crystalline polymer $(\mathrm{mPE})$ is higher than that of the more amorphous polymers $(\mathrm{mPE}-\mathrm{H}$ and $\mathrm{mPE}-\mathrm{O}$ ). Furthermore, and according to the authors, the increase in tensile strength at yield carries with it an increase in the material's rigidity. This would explain the trend observed with Young's modulus, with the more crystalline polymer (mPE) having higher elastic modulus than the amorphous ones (mPE$\mathrm{H}$ and $\mathrm{mPE}-\mathrm{O}$ ). In general, tensile strength at break follows the same trend. Therefore, a material with low crystallinity requires a low stress to become irreversibly strained. With respect to elongation, it is known that slippage and disentanglement would allow amorphous polymers to be deformed ${ }^{18}$. It is seen that when the amorphous proportion in the polymer increases, the percentage of elongation also increases. Also in Table 3, the decreased elongation observed after the polymers have been aged occurs because degradation takes place mostly in the amorphous phase ${ }^{19}$, scissioning polymer chains which then lack the capacity to elongate when the polymers undergo mechanical deformation. It is also noticed that copolymers of higher comonomer content aged $9136 \mathrm{~h}$ have higher elongation at break the than lower ones. It can be explained from the standpoint of ties molecules (TMs). These TMs in alpha olefine copolymers are co unit exclusion that disturbs the mechanism of regular chain folding which allow the crystallization process to occur, and so when the comonomer content increase the amorphous fraction increase allowing longer deformation. In molecular topology of semicristalline polymers the TMs are molecular interconnections which one are active elements for the stress transfer ${ }^{17}$ because allow the sample to carry large stresses to prevent higher strains ${ }^{20}$. The same author establishes that as comonomer content increase the concentration of TMs increase and then their tertiary hydrogens concentration in the amorphous phase. Due to the fact that tertiary hydrogen atom posses lower dissociation energy than other hydrogen atoms thus making it easier to convert to free radicals ${ }^{21}$, any molecular scission produced by the effect of thermal ageing will happen preferentially in this zone of the amorphous phase responsible of the stress transfer allowing the polymers with higher tertiary atoms concentration undergo higher strains which will produce longer elongations.

As Young's modulus, tensile strength at yield, and tensile stress at break depend mostly on the material's crystallinity, they are not affected when their magnitudes for the polymers with and without aging are compared.

\section{CONCLUSIONS}

Metallocene synthesis of homoethylene and ethylene-1-olefin copolymers allows narrower molecular weight distributions to be obtained. This fact makes possible the study of the influence of structure on degradation rate and final physical properties. It is clearly seen that branching in the main chain affects thermal stability, with comonomer size and content as two parameters to be studied. It is observed that lowering comonomer size as well as increasing comonomer content leads to an increase in autocatalytic oxidation rate when subjected to external thermal degradation. Tensile mechanical assays of copolymers showed the influence of comonomer content over percentage amorphous phase an then on their elongation properties. It is suggested that co units of tie molecules have twofold importance, i.e. increasing amorphous phase and prevents higher strain which would explain that when copolymers of higher comonomer content are subjected to thermal ageing they show longer elongation values. 


\section{ACKNOWLEDGEMENTS}

We acknowledge to CONICYT (Chile) through FONDAP Project 11980002, and Mr. Escudero scholarship. Also to Prof. Dr. Griselda Galland (UFRGS, Brasil) for NMR C13 analysis and Prof. Dr. Daniel Seraffini (USACH, Chile) for DSC analysis.

\section{REFERENCES}

1. R. Mülhaupt, Macromol Chem Phys, 204, 289, (2003)

2. R. Quijada, R. Scipioni, R. Mauler, G. Galland, M. Miranda, Polym Bull, 35, 299, (1995).

3. R. Quijada, A. Narvaez, R. Rojas, FM. Rabagliati, G. Barrera, RS. Mauler, R. Benavente, E. Pérez, JM. Pereña, A. Bello, Macromol. Chem. Phys., 200, 1306, (1999).

4. R. Quijada, J. Guevara, G. Galland, F. Rabagliati, J. Lopez-Majada, Polymer, 46, 1567, (2005).

5. P. Galli, G. Vecellio, J Polym Sci A: Polym Chem, 42, 396, (2004).

6. R. Benavente, E. Pérez, M. Yazdani-Pedram, R. Quijada, Polymer, 43, 6821, (2002).

7. EM. Hoàng, NS. Allen, CM. Liauw, E. Fontán, P. Lafuente, Polym Degrad Stab, 91, 1356, (2006)

8. S. Al-Malaika, X. Peng, H. Watson, Polym Degrad Stab, 91, 3131, (2006)
9. E. Pock, C. Kiss, A. Janecska, E. Epacher, B. Pukánsky, Polym Degrad Stab, 85, 1015, (2004)

10. B. Saha, AK. Maiti, AK. Ghoshal., Thermochim Acta, 444, 46, (2006).

11. L. Reich, S. Stivala, Elements of Polymer Degradation, McGraw-Hill Book Company, New York, 1971.

12. PA. Dilara, D. Briassoulis, Polym Test, 17, 549, (1998).

13. IM. Ward, DW. Hadley, An Introduction to the Mechanical Properties of Solid Polymers, John Wiley \& Sons Ltd, 2004.

14. M. Bertoldo, S. Bronco, C. Cappelli, T. Gragnoli, L. Andreotti, J Phys Chem $B, \mathbf{1 0 7}, 11880,(2003)$.

15. S. Bensanson, J. Minick, A Moet, S. Chum, A. Hiltner, E. Baer, J Polym Sci B: Polym Phys, 34, 1301, (1996).

16. O. Chiantore, S. Tripodi, C. Sarmoria, E. Vallés, Polymer, 42, 3981, (2001).

17. R. Seguela, J Polym Sci B: Polym Phys, 43, 1729, (2005).

18. C. Li Pi Shan, JBP. Soares, A. Penlidis, Polymer, 43, 767-773, (2002).

19. PA. Dilara, D. Briassoulis, J Agric Engng Res, 76, 309, (2000).

20. K. Jordens, GL. Wilkes, J. Janzen, DC. Rohlfing, MB. Welch, Polymer, 41, 7175, (2000).

21. RK. Rowe, HP. Sangman, Geotextiles and Geomembranes, 20, 77, (2002). 